\title{
Alterações vasculares na coróide de ratos diabéticos tratados e não tratados
}

\author{
Choroidal vessels alterations in treated and untreated diabetic rats
}

\author{
Antonio Carlos Lottelli Rodrigues ${ }^{1}$ \\ Silvana Artioli Schellini ${ }^{2}$ \\ César Tadeu Spadela ${ }^{3}$ \\ Elisa Aparecida Gregório ${ }^{4}$ \\ Carlos Roberto Padovani ${ }^{5}$
}

Trabalho realizado no Laboratório Experimental da Faculdade de Medicina da Universidade Estadual Paulista "Júlio Mesquita Filho" - UNESP - Botucatu (SP) - Brasil.

${ }^{1}$ Professor Doutor do Departamento de Oftalmologia, Otorrinolaringologia e Cirurgia de Cabeça e Pescoço da Faculdade de Medicina da Universidade Estadual Paulista "Júlio Mesquita Filho" - UNESP - Botucatu (SP) - Brasil.

${ }^{2}$ Livre- Docente do Departamento de Oftalmologia, Otorrinolaringologia e Cirurgia de Cabeça e Pescoço da Faculdade de Medicina da UNESP - Botucatu (SP) Brasil.

${ }^{3}$ Livre-Docente do Departamento de Cirurgia da Faculdade de Medicina da UNESP - Botucatu (SP) - Brasil.

${ }^{4}$ Professora Titular do Departamento de Morfologia do Instituto de Biociências da UNESP - Botucatu (SP) Brasil.

5 Professor Titular do Departamento de Bioestatística, Instituto de Biociências da UNESP - Botucatu (SP) Brasil.

Endereço para correspondência: Antonio Carlos Lottelli Rodrigues. Faculdade de Medicina de Botucatu UNESP - Distrito de Rubião Júnior $\mathrm{s} / \mathrm{n}^{\circ}$ - Botucatu (SP) CEP 18618-970

E-mail: eye_acr@fmb.unesp.br

Recebido para publicação em 05.08.2006

Aprovação em 26.02.2007

\begin{tabular}{l} 
RESUMO \\
\hline Objetivo: Conhecer os efeitos do diabetes e o impacto de seu tratamento \\
medicamentoso em curto e longo prazo sobre os vasos da coróide e \\
membrana de Bruch. Métodos: Foram estudados 30 ratos Wistar, divididos \\
em 3 grupos experimentais: grupo controle (GC), grupo diabético (GD) e \\
grupo diabético tratado (GT), estudados 1 mês (momento M M $_{1}$ ) 12 meses \\
(momento $\mathrm{M}_{2}$ ) após o início do experimento. O diabetes foi induzido por \\
aloxana endovenosa, na dose de 42 mg/kg. O GT foi tratado com hipogli- \\
cemiante oral (acarbose) e insulina subcutânea. Após o sacrifício, os olhos \\
foram preparados para exame ao microscópio eletrônico de transmissão, \\
interessando a ultra-estrutura da membrana de Bruch e os vasos da \\
coróide. Resultados: O exame ultra-estrutural da coróide dos ratos diabé- \\
ticos mostrou depósitos na membrana de Bruch, acúmulo de vesículas, \\
glicogênio e corpos densos no citoplasma das células endoteliais. O grupo \\
mais afetado foi de ratos diabéticos de 12 meses (GDM2). Os animais com \\
menor intensidade de alterações foram os ratos tratados por 12 meses \\
(GTM2). Conclusão: Os ratos diabéticos desenvolveram alterações dege- \\
nerativas na membrana de Bruche vasos da coróide. Estas alterações foram \\
mais evidentes nos animais submetidos à doença crônica, mas também \\
ocorreram agudamente. O tratamento a curto prazo não foi capaz de evitar \\
os processos degenerativos. A longo prazo, o tratamento inibiu a progres- \\
são destes processos.
\end{tabular}

Descritores: Coróide/irrigação sanguínea; Membrana de bruch; Diabetes mellitus experimental; Ratos Wistar; Aloxana; Insulina; Acarbose

\section{INTRODUÇ̃̃̃OO}

A coróide possui importante papel na nutrição e na manutenção da integridade retiniana ${ }^{(1)}$.

O termo coroidopatia diabética é usado como referência a alterações da coróide decorrentes do diabetes ${ }^{(2)}$. Estas alterações são muito semelhantes as que ocorrem na retinopatia ${ }^{(2-3)}$ e tem importante papel na gênese da retinopatia ${ }^{(4)}$ e da maculopatia diabética ${ }^{(5)}$, podendo ser encontradas mesmo na ausência de retinopatia detectável ${ }^{(6)}$. Os principais fatores de risco para coroidopatia diabética são a severidade da retinopatia e o grau de controle da glicemia $^{(7)}$.

Com relação a estudos experimentais, há animais que desenvolvem diabetes espontaneamente, como o rato BB Wistar, o cão Keeshond, a macaca Nigra, algumas raças de hamsters e de camundongos. A doença pode, ainda, ser induzida em animais, de várias maneiras: estresse, infecções, toxinas, pancreatectomia; lesões do sistema nervoso central; uso de hormônios antiinsulínicos; exposição à hidrocortisona ou ACTH; indução 
por vírus e uso de agentes beta-citotóxicos como o aloxano e a estreptozotocina.

Estudos experimentais da coróide de ratos diabéticos demonstram espessamento da membrana basal ${ }^{(8-9)}$ e diminuição nos sítios aniônicos desta membrana nos vasos da corioca$\operatorname{pilar}^{(9)}$, proliferação de células endoteliais ${ }^{(8)}$, aceleração dos seus processos degenerativos senescentes ${ }^{(10)}$ e outras alterações hemodinâmicas e de barreira na microcirculação ${ }^{(11)}$. Estas alterações são mais acentuadas quanto maior o nível glicêmico, mas também estão presentes em diabetes leve e de maneira desproporcionalmente mais acentuadas do que o suposto pelos níveis glicêmicos ${ }^{(11)}$. Podem ser irreversíveis ${ }^{(12)}$ ou até mesmo aumentar as áreas de destruição quando o nível glicêmico normal é reinstalado ${ }^{(13)}$.

Hoje, o impacto do controle glicêmico sobre as alterações na retina está bem estabelecido ${ }^{(14)}$.

No entanto, estudos sobre os efeitos deste controle sobre a coróide são poucos e voltados para a resposta aos tratamentos cirúrgicos ${ }^{(13)}$.

O presente estudo tem o objetivo de comparar a curto e a longo prazo, os efeitos da doença e a repercussão do tratamento medicamentoso sobre a ultra-estrutura da coróide.

\section{MÉTODOS}

Foram utilizados 30 ratos da raça Wistar, de ambos os sexos, com idade de 3 meses, pesando de 200 a 300 gramas. Os animais foram divididos por sorteio, em 3 grupos experimentais: 10 ratos normais (GC), 10 ratos diabéticos que não receberam tratamento (GD) e 10 ratos diabéticos que receberam tratamento com hipoglicemiante oral, associado a insulinoterapia (GT).

Para indução do diabetes, os ratos receberam injeção endovenosa de solução aquosa de aloxana 2\% (2,4,5,6 tetraoxohexahydropyrimidine), em dose única de $42 \mathrm{mg} / \mathrm{kg}$ de peso corporal.

Após a indução da doença, os animais foram seguidos por 15 dias para a constatação do estado de diabetes grave. Depois disso, iniciou-se a pesquisa.

Os animais foram sacrificados em dois momentos experimentais: 1 mês após o início do experimento $\left(M_{1}\right)$ e 12 meses após $\left(\mathrm{M}_{2}\right)$, sendo sacrificados 5 animais de cada grupo, em cada momento experimental.

A análise ultra-estrutural da coróide foi realizada por microscopia eletrônica de transmissão, nos 30 animais, com atenção à membrana de Bruch e aos vasos.

Os ratos dos grupos GC e do GD receberam ração Nuvilab CR1 (Nuvital Nutrientes e Produtos Veterinários Ltda. - PR). Os do GT receberam hipoglicemiante oral (acarbose - Bay G-5421 - Cia. Bayer do Brasil) adicionado à dieta habitualmente fornecida aos animais, e injeções de insulina porcina monocomponente, de ação prolongada Monotard R (40.000 U/ml Laboratório Nova Indústria Ltda.).

Quinzenalmente foram realizadas avaliações clínico-laboratoriais, quando os animais foram colocados em gaiolas metabólicas individuais, durante 24 horas.
No momento do sacrifício, os animais receberam dose letal de pentobarbital sódico a 3\% (30 mg/ $\mathrm{kg}$ de peso corporal) endovenoso. Após anestesia, foi feita toracotomia, seguida de punção cardíaca para obtenção de amostras de sangue destinadas aos exames laboratoriais.

Os olhos foram enucleados imediatamente após o sacrifício, sendo preparados para exame ao microscópio eletrônico de transmissão.

\section{RESULTADOS}

\section{GCM1}

A coróide de ratos é constituída por membrana de Bruch, coriocapilar formada por vasos finos e fenestrados, localizados logo abaixo desta membrana e vasos mais calibrosos com característica de vênulas ou arteríolas, mergulhados no estroma e localizados abaixo da coriocapilar (Figura 1A e B).

\section{GCM2}

Comparando-se a ultra-estrutura da membrana de Bruch de ratos de $\mathrm{GCM}_{1}$ com os de $\mathrm{GCM}_{2}$, não se observaram alterações.

Observaram-se vasos da coriocapilar e estromais com a mesma constituição da observada nos ratos do $\mathrm{GCM}_{1}$, exceto pela aparente maior quantidade de glicogênio, vesículas citoplasmáticas e corpos densos nas células endoteliais, tanto na região próxima ao lúmen vascular, quanto na porção próxima ao estroma (Figura 1C e D).

\section{GDM1}

A ultra-estrutura da membrana de Bruch de ratos $\mathrm{GDM}_{1}$ foi semelhante à observada em GCM $_{1}$. Em algumas regiões observou-se a presença de substância amorfa, homogênea, eletrondensa, que, em algumas áreas, ocupou toda sua espessura (Figura 2A).

Observaram-se vasos com a mesma constituição dos observados em ratos do $\mathrm{GCM}_{1}$. Com relação à quantidade de vesículas citoplasmáticas, assim como de glicogênio livre, houve aumento da quantidade, quando comparados com animais normais de GCM1 ou de GCM2, tanto em células endoteliais, como nos pericitos. Notou-se, também, aumento da quantidade de corpos densos no citoplasma das células endoteliais e pericíticas (Figura 2B, C e D).

As membranas basais (MB) das células endoteliais e também dos pericitos apresentaram espessura aumentada, principalmente nos vasos de maior calibre (Figura 2B, C e D).

\section{GDM2}

A membrana de Bruch de ratos diabéticos de $\mathrm{GDM}_{2}$ apresentou, em várias regiões, a presença de espaços eletrontransparentes (Figura 2E). Em outras regiões, foi encontrada substância amorfa, eletrondensa entre as camadas de fibras colágenas e elásticas e espessamento da quinta camada (Figura 2F).

Observaram-se vasos com estrutura semelhante aos encontrados nos ratos do $\mathrm{GCM}_{1}$. Porém, nestes animais, foi 
possível observar, maior quantidade de vesículas citoplasmáticas, glicogênio livre e de corpos densos do que o encontrado nos animais dos demais grupos, tanto nas células endoteliais, como nos pericitos (Figura $2 \mathrm{G}$ ).

A MB também esteve mais espessa nos animais deste grupo, principalmente a $\mathrm{MB}$ dos vasos de maior calibre. Observaram-se espaços entre as células endoteliais e pericitos totalmente preenchidos pela MB espessada (Figura $2 \mathrm{G}$ ).

\section{GTM1}

Observaram-se na membrana de Bruch de ratos GTM, algumas áreas contendo substância amorfa, homogênea, eletrondensa, entre a segunda, terceira e quarta camadas, semelhantes às observadas nos ratos do GDM . A densidade deste material, no entanto, foi maior que o daquele observado no $\mathrm{GDM}_{1}$ (Figura 3A, B e C).

Os vasos da coróide de animais diabéticos tratados mostraram-se semelhantes aos de ratos diabéticos não tratados de mesma idade (GDM1). No entanto, maior número de vesículas citoplasmáticas, glicogênio e corpos densos foram observados, quando comparados ao GDM1, tanto em células endoteliais como em pericitos. Além disso, em algumas células endoteliais, existiram vacúolos contendo material heterogêneo e espaços eletrontrasparentes (Figura 3B, C e D).

\section{GTM2}

A membrana de Bruch de ratos $\mathrm{GTM}_{2}$ foi semelhante à observada no GTM 1 (Figura 3E e F).

As alterações presentes nos vasos dos ratos tratados de $\mathrm{GTM}_{2}$ foram menos intensas que as existentes nos animais do $\mathrm{GDM}_{2}$, podendo-se considerá-las semelhantes às encontradas nos ratos do $\mathrm{GDM}_{1}$ (Figura 3E, G e H).

\section{DISCUSSÃO}

\section{Membrana de Bruch}

O presente estudo mostra depósitos de substância amorfa, homogênea e eletrondensa, em algumas regiões da membrana de Bruch.

A própria senescência pode causar espessamento desta membrana, sugerindo-se que este espessamento possa ser decorrente do depósito progressivo de material provindo da coriocapilar, epitélio pigmentar retiniano ou alterações in situ.

Em humanos diabéticos, são descritas degenerações da membrana de Bruch $^{(15)}$, assim como presença de depósitos ${ }^{(16)}$ e espessamento da quinta camada ${ }^{(8)}$. As drusas também estão presentes na membrana de diabéticos, o que se observou por meio da angiografia fluorescente ${ }^{(17)}$.

Estudos em ratos demonstram diminuição dos sítios aniônicos da primeira camada ${ }^{(9)}$, espessamentos focais e difusos, com desarranjo de orientação das fibras colágenas, ou uniformes às custas da condensação do colágeno ${ }^{(18)}$.

Os depósitos foram discretos nos ratos do GDM1, mas importantes no GDM2, onde também foram observados espa- ços eletrontransparentes por entre as camadas e espessamento da quinta camada. Estas alterações podem ser atribuídas ao diabetes, já que não foram encontradas no grupo controle.

Em relação ao tratamento a curto prazo, não houve diminuição destes depósitos, parecendo ter ocorrido até efeito maléfico, pois os depósitos observados em algumas regiões do GTM1 eram de densidade nitidamente maior, que os observados no GDM1.

A longo prazo, entretanto, o tratamento mostrou seu benefício. As alterações encontradas no GTM2 podem ser comparadas às de GDM1. Assim a continuidade do tratamento parece ter revertido a piora inicial e impedido sua progressão.

Estes achados têm precedentes na literatura. Autores descreveram extensas áreas de destruição na retina e coróide de ratos diabéticos induzidos, submetidos a transplante de ilhotas pancreáticas ${ }^{(13)}$. Os autores atribuíram estas alterações a processos isquêmicos na microcirculação, questionando-se o retorno a níveis glicêmicos normais como inicialmente maléfico a estas estruturas.

Entretanto, existe apenas aquela referência abordando as alterações na membrana de Bruch após tratamento ${ }^{(13)}$, o que torna necessário novos estudos para elucidar estes achados e responder a esta pergunta.

\section{Alterações vasculares}

Em relação aos vasos da coróide diabética, os estudos existentes mostram de forma variável, alterações muito semelhantes àquelas encontradas na retinopatia diabética ${ }^{(2)}$. No entanto, os vasos da coróide não possuem a mesma estrutura que os vasos retinianos, uma vez que são vasos fenestrados e os retinianos, contínuos.

Ratos diabéticos não desenvolvem as mesmas lesões vasculares que os homens. Estes roedores parecem ter resistência maior ao desenvolvimento da vasculopatia. A neovascularização, nunca foi demonstrada na retina destes animais ${ }^{(19)}$, apesar do aumento nos níveis de fator de crescimento endotelial vascular (VEGF), demonstrado nas fibras do nervo óptico, epitélio pigmentar retiniano e coróide de ratos GotoKakizaki $^{(20)}$.

No entanto, estes animais podem apresentar várias alterações vasculares na retina e na coróide, como espessamento da MB, perda de pericitos, vasodilatação, atrofia capilar, microaneurismas $^{(21-22)}$ e são usados como modelos experimentais devido, principalmente, ao curto ciclo de vida destes animais.

As alterações vasculares encontradas no presente estudo ocorreram basicamente nas organelas citoplasmáticas das células endoteliais e pericitos e na MB destas células.

No citoplasma, observaram-se acúmulo de vesículas, corpos densos e glicogênio livre.

O significado do aumento das vesículas é desconhecido. Sabe-se que as vesículas citoplasmáticas são relacionadas ao transporte de substâncias, sendo responsáveis, pela remoção de detritos celulares e moléculas tóxicas do interstício para o lúmen vascular, o aumento das mesmas poderia estar relacionado à exacerbação destes processos no diabetes. 


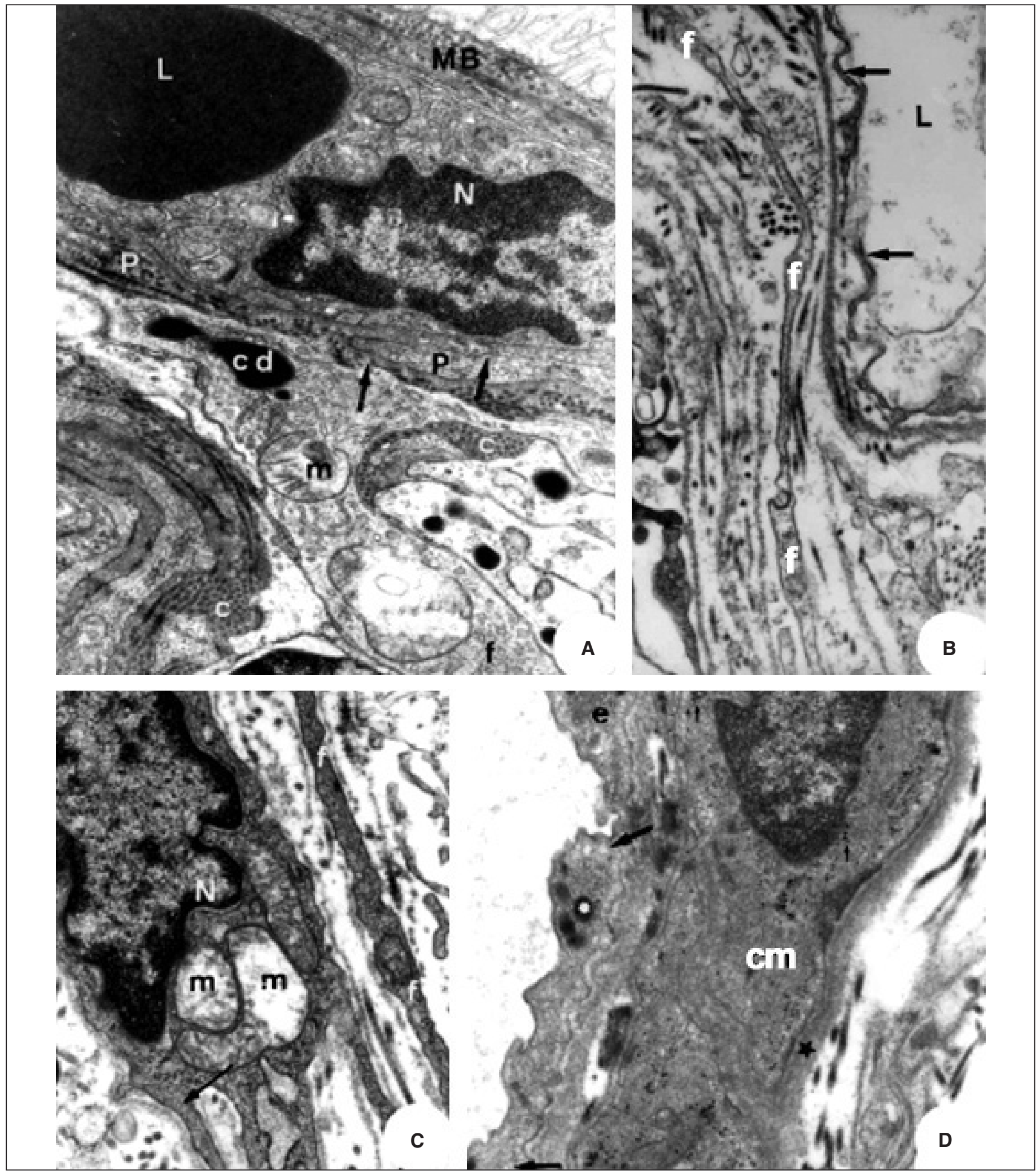

Figura 1 - A: Vasos e estroma da coróide de rato do GCM1. Células endoteliais, envoltas por pericitos (P). Núcleo (N) alongado da célula endotelial. Luz da coriocapilar com eritrócito (L). Membrana basal entre as células endoteliais e os pericitos (seta), assim como também entre os pericitos e os tecidos circunvizinhos. Fibroblastos (f) com alguns corpos densos (cd). Fibras colágenas (C) cortadas em diversos sentidos de orientacão. Membrana de Bruch (MB) (x 27.500); B: Vasos e estroma da coróide de rato do GCM1. Observar as fenestrações (seta) das células endoteliais da coriocapilar. Luz da coriocapilar (L). Fibroblasto estromal (f) (x 27.500); C: Vasos da coróide de rato do GDM1. Observar a grande quantidade de vesículas (seta) (x 27.500); D: Vasos da coróide de rato do GDM1. Células endoteliais (e) com aumento de vesículas (seta). Pericitos (p) com aumento de vesículas e membranas basais espessadas (asterisco) (x 27.500). 


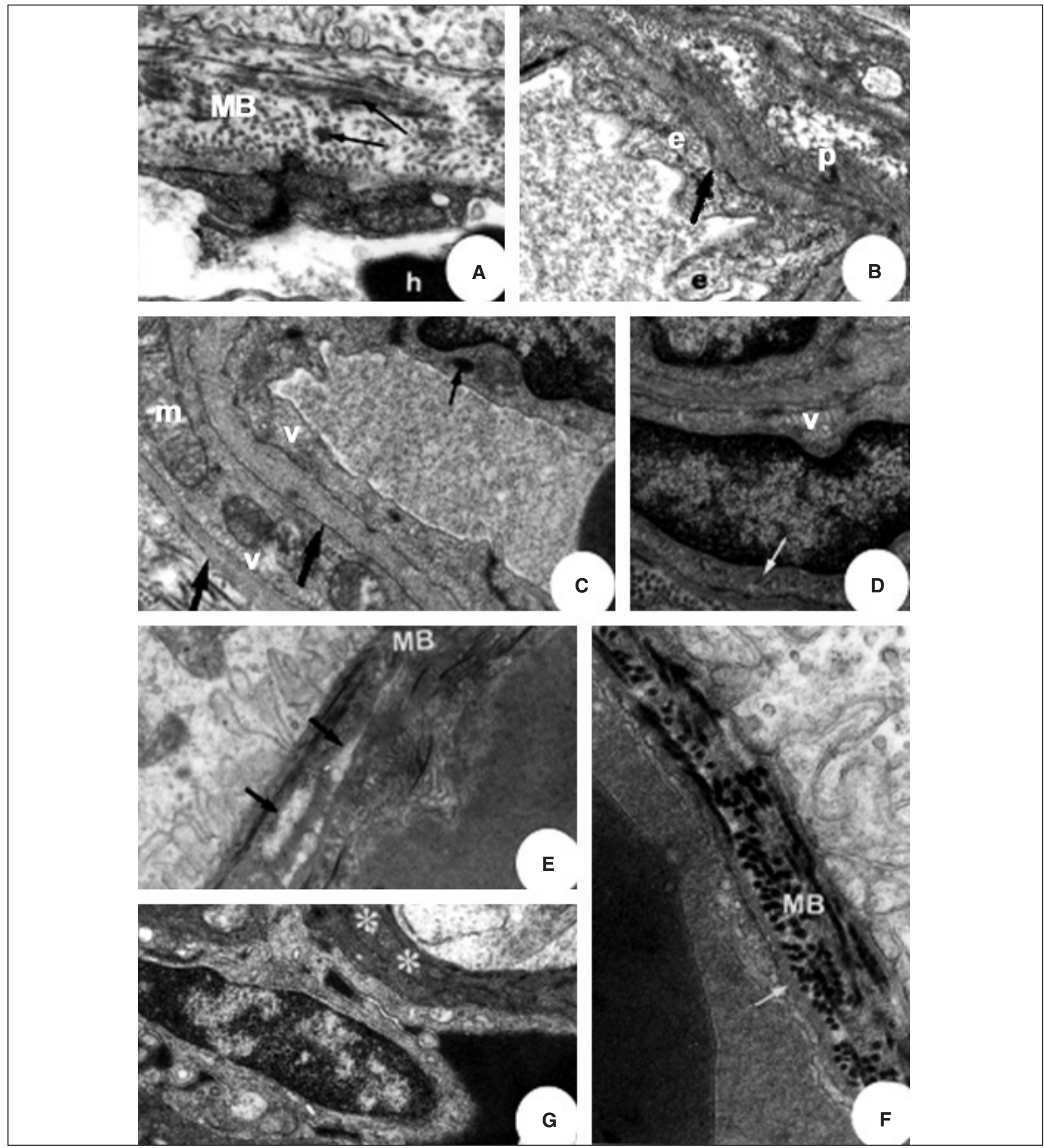

Figura 2 - A: Vasos da coróide de rato do GDM2. Grande espessamento da membrana basal vascular (asterisco) (x 27.500); B: Transição entre o epitélio pigmentar (EP) e a coróide de ratos do GTM1. Membrana de Bruch (MB) e vaso da coriocapilar com hemácia (H) (x 17.000); C: Vaso da coróide de rato do GTM1. O citoplasma da célula endotelial do vaso da coriocapilar apresenta espaços eletrontransparentes (estrela) e corpos densos (seta larga). Membrana de Bruch (MB). Hemácia (H). Pericito com acúmulo de glicogênio (seta fina) e corpos densos (seta larga) (x 23.000); D: Membrana de Bruch e estroma da coróide de rato do GTM1. Presença de substâncias eletrondensas na membrana de Bruch e entre fibras colágenas do estroma (seta) (x 17.000); E: Estroma da coróide de rato do GTM2. Presença de grandes espaços eletrontransparentes (estrela) (x 23.000); F: Estroma da coróide de rato do GTM2. Acúmulo de glicogênio (asterisco) e corpos densos (seta) em fibroblasto estromal (x 23.000); G: Vaso e estroma da coróide de rato do GTM12. Célula endotelial com núcleo picnótico e citoplasma rarefeito (seta larga). Presença de espaço eletrontransparente no estroma (estrela) (x 17.000). 


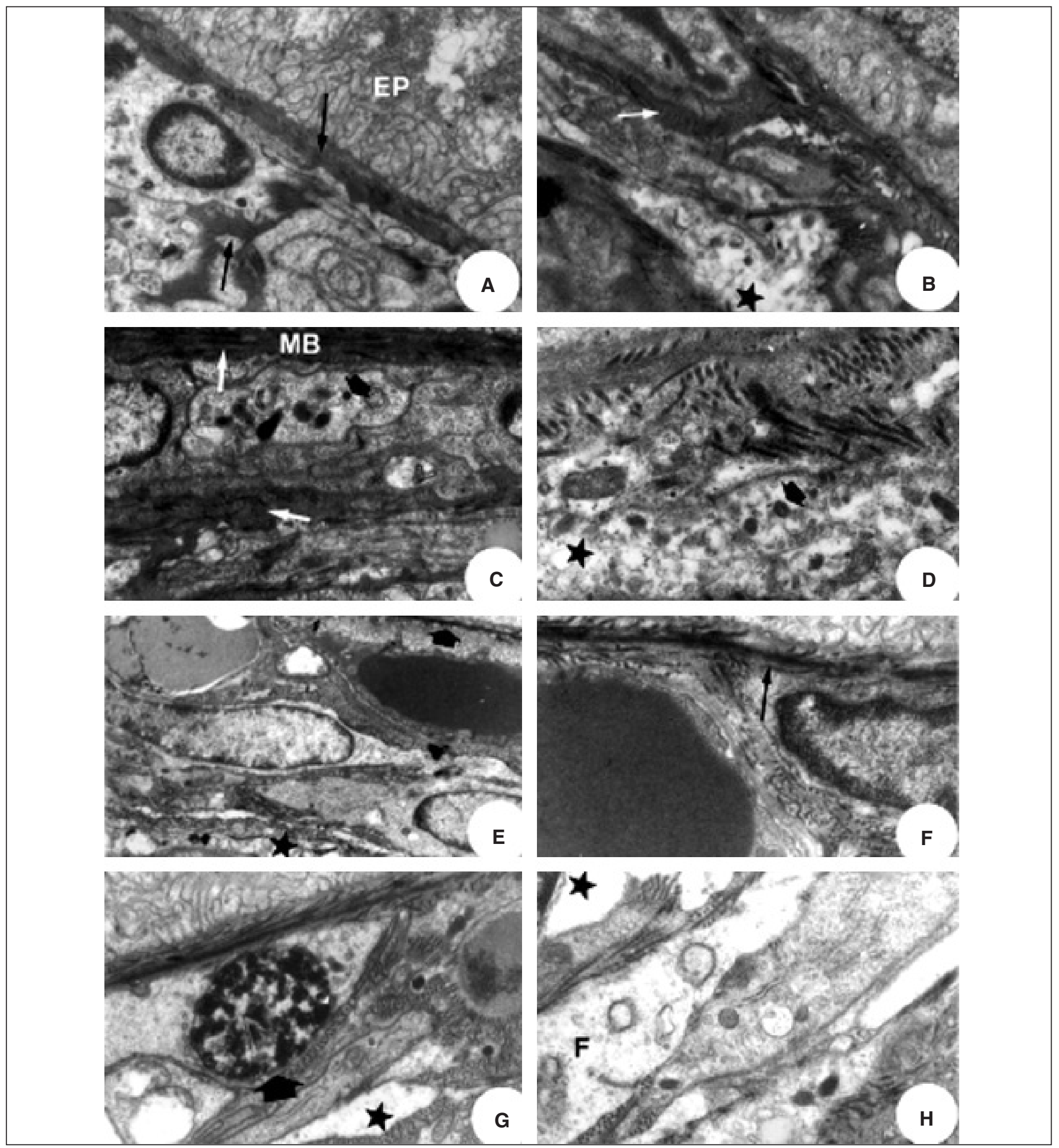

Figura 3 - A: Membrana de Bruch e estroma da coróide de rato do GTM1. Presença de substâncias eletrondensas na membrana de Bruch e entre fibras colágenas do estroma (seta) (x 17.000); B: Vasos e estroma da coróide de ratos do GTM1. Presença de espacos eletrontransparentes no estroma (estrela) e depósitos de substância eletrondensa por entre as fibras colágenas estromais (seta) ( $x$ 17.000); C: Membrana de Bruch (MB) e vasos da coróide e estroma de rato do GTM1. Depósitos eletrondensos na membrana de Bruch e fibras colágenas do estroma (seta fina). Presença de vacúolos com material heterogenia e corpos densos no citoplasma de diferentes células (seta grossa) (x17.000); D: Estroma da coróide de rato do GTM1. Fibroblasto com citoplasma rarefeito (estrela) e grande número de corpos densos (seta) (x 23.000); E: Aspecto geral da coróide de rato do GTM12. Membrana de Bruch (seta). Espaços eletrontransparentes no estroma (estrela) (x 7.750); F: Membrana de Bruch da coróide de rato do GTM2. Presença de depósito eletrondenso densa na terceira camada (seta) (x 23.000); G: Vaso e estroma da coróide de rato do GTM12. Célula endotelial com núcleo picnótico e citoplasma rarefeito (seta larga). Presença de espaço eletrontransparente no estroma (estrela) (x 17.000); H: Estroma da coróide de rato do GTM2. Fibroblasto com citoplasma rarefeito $(F)$ e espaço eletrontransparente (estrela) (x 23.000). 
Outros acúmulos citoplasmáticos, como o de corpos densos, também relacionados à remoção de detritos intracelulares poderiam novamente, refletir a degeneração das organelas.

O acúmulo de vesículas, corpos densos e glicogênio livre, foi observado nos ratos do GCM2. Assim, existe consistência para supor que possam estar ocorrendo processos degenerativos relacionados com a própria senescência ${ }^{(19)}$. No entanto, a presença destas organelas, em maior quantidade nos animais diabéticos (GDM1 e ainda maior no GDM2), pode ser indício de que o estado diabético esteja relacionado com a exacerbação dos processos degenerativos. Em relação aos grupos tratados, assim como ocorreu na membrana de Bruch, o tratamento a curto prazo não foi capaz de impedir com que ocorressem alterações e o acúmulo das organelas associadas ao mecanismo de "limpeza celular" foi ainda maior no GTM1, que no GDM1, concordando mais uma vez com a literatura ${ }^{(11)}$.

A longo prazo, no entanto, o tratamento mostrou seu beneficio através dos achados no GTM2, quando a quantidade de vesículas, corpos densos e glicogênio, foi semelhante ao que se encontrou no GDM1. Assim, os achados ultra-estruturais permitem supor que a continuidade do tratamento do diabetes não só freou os processos degenerativos, mas também foi capaz de reverter alterações inicialmente causadas.

Os acúmulos de vesículas e corpos densos no citoplasma das células endoteliais, encontrados neste estudo, concordam com os achados da literatura no que diz respeito ao processo de degeneração acelerada desses $\operatorname{vasos}^{(10,23)}$.

$\mathrm{O}$ espessamento da MB dos vasos, principalmente nos de maior calibre, foi demonstrado nos ratos diabéticos. Este espessamento foi maior no GDM2, que nos demais grupos diabéticos (GDM1, GTM1 e GTM2), nos quais houve semelhança.

$\mathrm{O}$ espessamento da parede dos vasos da coróide, às custas de aterosclerose e depósitos PAS (ácido periódico de Schiff) positivos, foram evidentes em estudos histológicos ${ }^{(3)}$ e de microscopia eletrônica de varredura ${ }^{(4)}$ e indiretamente demonstrados pela detecção de retardo de perfusão à angiofluoresceinografia $^{(17)}$. Além do espessamento da MB da coriocapilar, foi demonstrado diminuição dos sítios aniônicos nesta membra$\mathrm{na}^{(9)}$. Posteriormente, demonstrou-se também, que estes sítios estavam diminuídos nas próprias células endoteliais da coriocapilar $^{(23)}$. Eles são importantes para regulação da passagem de proteínas através da membrana de Bruch. Essa barreira aniônica é formada pelas proteoglicanas que, sabidamente, encontramse reduzidas em tecidos diabéticos como, por exemplo, na lâmina basal do glomérulo renal ${ }^{(23)}$.

Utilizando ratos diabéticos e hipertensos, autores encontraram proliferação aumentada de células endoteliais que levaram a estreitamento na luz vascular; no entanto, este achado foi mais evidente nos ratos que também eram hipertensos, animais que apresentavam, portanto, superposição de fatores agressores $^{(8)}$. A hipercelularidade vascular também é achada de literatura ${ }^{(4)}$ que não foi observada no presente estudo.

Outros estudos feitos em vasos coroidais diabéticos não mostraram alteração no tamanho do capilar ou na distribuição do número de pericitos ${ }^{(21)}$. Estes autores realizaram, inclusive, medidas morfométricas da área luminal, espaços intervasculares, número de fenestrações voltadas para o epitélio pigmentar retiniano e para o estroma, não encontrando diferença estatisticamente significativa entre ratos normais e diabéticos. Assim, apesar de terem observado alterações ultra-estruturais, as medidas morfométricas não diferiram entre ratos normais e diabéticos.

Talvez estas aparentes discrepâncias entre os estudos possam ser explicadas pela sugestão de que, não só o processo de degeneração, mas também o de renovação destes vasos, estejam aumentados $^{(23)}$. Ou seja, as alterações vasculares são dinâmicas, havendo chance de lesão e de reparação, o que pode permitir diferentes observações.

\section{CONCLUSÃO}

Diante dos resultados obtidos no presente estudo, foi possível concluir que os ratos normais M2 apresentaram discretas alterações em componentes citoplasmáticos das células endoteliais, provavelmente por processo de senilidade.

Os ratos diabéticos apresentaram alterações ultra-estruturais na coróide, ocorrendo na membrana de Bruch e nos vasos. Estas alterações foram mais evidentes nos animais submetidos à doença crônica, mas também ocorreram agudamente.

O tratamento do diabetes a curto prazo não impediu os processos degenerativos e aparentemente até os exacerbou, tanto na membrana de Bruch, como nos vasos da coróide. A longo prazo, o tratamento mostrou-se efetivo, inibindo a progressão dos processos degenerativos.

\section{ABSTRACT}

Purpose: To evaluate the diabetic alterations and the impact of short and long-term medical treatment on them. Methods: Thirty Wistar rats were divided into 3 groups: control (GC), diabetic (DG), and treated diabetic (TG) and the observations were made 1 month $\left(\mathrm{M}_{1}\right)$ and 12 months $\left(\mathrm{M}_{2}\right)$ after diabetes induction. Diabetes was induced by intravenous alloxan $(42 \mathrm{mg} / \mathrm{kg})$. The treated group received acarbose orally and insulin by subcutaneous injection. Eyes were prepared for transmission electron microscopy, specifically for ultrastructure of the Bruch membrane and choroidal vessels. Results: Ultrastructural examination of the diabetic rat coroid showed deposits in the Bruch membrane and accumulation of vesicles, glycogen and dense bodies in endothelial cell cytoplasm. The most affected group was that of the diabetics on month 12 (GDM2). The treated diabetics showed the least alterations on month 12 (GTM2). Conclusion: Diabetic rats develop degenerative alterations in the Bruch membrane and choroidal vessels. These alterations are more evident in animals submitted to chronic disease, but they are also present in acute disease. Degenerative processes were not avoided with short-term treatment. Long-term treatment inhibited the progress of these processes. 
Keywords: Choroid/blood supply; Bruch membrane; Diabetes mellitus, experimental; Rats, Wistar; Alloxan; Insulin; Acarbose

\section{REFERÊNCIAS}

1. May CA, Horneber M, Lutjen-Drecoll E. Quantitative and morphological changes of the choroid vasculature in RCS rats and their congenic controls. Exp Eye Res. 1996;63(1):75-84.

2. Saracco JB, Gastaud P, Ridings B, Ubaud CA. [Diabetic choroidopathy]. J Fr Ophtalmol. 1982;5(4):231-6. French.

3. Hidayat AA, Fine BS. Diabetic choroidopathy. Light and electron microscopic observations of seven cases. Ophthalmology. 1985;92(4):512-22.

4. Fryczkowski AW. Vascular casting and scanning electron microscopy in diabetes. Scanning Microsc. 1987;1(2):811-6.

5. Takamine Y. [Disorders of choroidal circulation in diabetic maculopathy]. Nippon Ganka Gakkai Zasshi. 1998;102(8):487-94. Japanese.

6. Fukushima I, McLeod DS, Lutty GA. Intrachoroidal microvascular abnormality: a previously unrecognized form of choroidal neovascularization. Am J Ophthalmol. 1997;124(4):473-87.

7. Shiragami C, Shiraga F, Matsuo T, Tsuchida Y, Ohtsuki H. Risk factors for diabetic choroidopathy in patients with diabetic retinopathy. Graefes Arch Clin Exp Ophthalmol. 2002;240(6):436-42.

8. Hori S, Nishida T, Mukai Y, Pomerov M, Mukai N. Ultrastructrual studies on choroidal vessels in streptozotocin-diabetic and spontaneously hypertensive rats. Res Commun Chem Pathol Pharmacol. 1980;29(2):211-28.

9. Caldwell RB, Slapnick SM, McLaughlin BJ. Decreased anionic sites in Bruch's membrane of spontaneous and drug-induced diabetes. Invest Ophthalmol Vis Sci. 1986;27(12):1691-7.

10. Rodrigues AC, Schellini SA, Gregorio EA, Spadella CT, Padovani CR. Choroidal vasculature in diabetic rats. J Submicrosc Cytol Pathol. 2004;36(34):327-31.

11. Pugliese G, Tilton RG, Speedy A, Chang K, Santarelli E, Province MA, et al. Effects of very mild versus overt diabetes on vascular haemodynamics and barrier function in rats. Diabetologia. 1989;32(12):845-57.
12. Pugliese G, Tilton RG, Chang K, Speedy A, Province MA, Eades D, et al. Effects of islet isografts on hemodynamic and vascular filtration changes in diabetic rats. Diabetes. 1990;39(3):323-32.

13. Kimura S, Aoyagi K, Ogawa Y, Suzuki K, Mizutani H, Yoshimoto H, et al [Retinal ischemic changes in diabetic rats treated with pancreatic islet transplantation beneath the renal capsule]. Nippon Ganka Gakkai Zasshi. 1993;97 (7):800-11. Japanese.

14. The effect of intensive treatment of diabetes on the development and progression of long-term complications in insulin-dependent diabetes mellitus. The Diabetes Control and Complications Trial Research Group. N Engl J Med. 1993;329(14):977-86

15. McLeod DS, Lefer DJ, Merges C, Lutty GA. Enhanced expression of intracellular adhesion molecule-1 and P-selectin in the diabetic human retina and choroid. Am J Pathol. 1995;147(3):642-53.

16. Cao J, McLeod S, Merges CA, Lutty GA. Choriocapillaris degeneration and related pathologic changes in human diabetic eyes. Arch Ophthalmol. 1998; 116(5):589-97.

17. Freyler H, Prskavec F, Stelzer N. [Diabetic choroidopathy - a retrospective fluorescein angiography study. Preliminary report]. Klin Monatsbl Augenheilkd. 1986;189(2):144-7. German.

18. Jorge EC, Trindade JCS. Retinopatia diabética: estudo ultraestrutural e morfométrico do epitélio pigmentar de ratos normais e aloxônicos. Rev Bras Oftalmol. 1997;56(7):461-78.

19. Schellini SA, Gregorio EA, Padovani CR, Spadella CT, Moraes-Silva MR. Ultrastructural and morphometric aspects of ageing in the retinal capillaries of rats. J Submicrosc Cytol Pathol. 1997;29(2):275-80.

20. Sone H, Kawakami Y, Okuda Y, Sekine Y, Honmura S, Matsuo K, et al Ocular vascular endothelial growth factor levels in diabetic rats are elevated before observable retinal proliferative changes. Diabetologia. 1997;40(6):726-30.

21. Tilton RG, LaRose LS, Kilo C, Williamson JR. Absence of degenerative changes in retinal and uveal capillary pericytes in diabetic rats. Invest Ophthalmol Vis Sci. 1986;27(5):716-21.

22. Schellini SA, Gregorio EA, Spadella CT, Machado JL, de-Moraes-Silva MA Muller cells and diabetic retinopathy. Braz J Med Biol Res. 1995;28(9):977-80.

23. Caldwell RB, Fitzgerald ME. The choriocapillaris in spontaneously diabetic rats. Microvasc Res. 1991;42(3):229-44.

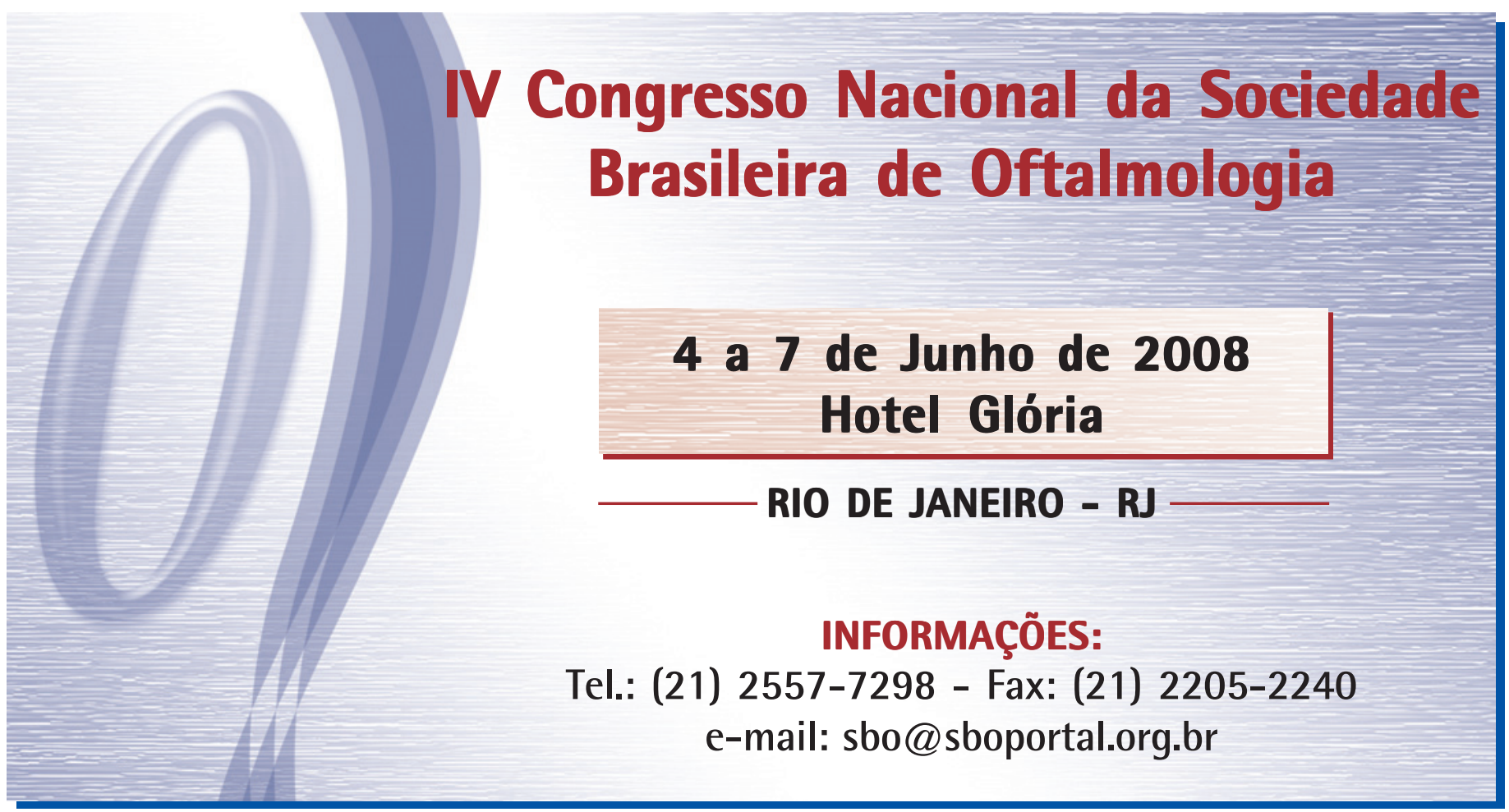

